\title{
POLYHYDROXYALKANOATES: ADVANCES IN THE SYNTHESIS OF SUSTAINABLE BIO-PLASTICS
}

\author{
SHREYA SHAH and ANIL KUMAR* \\ School of Biotechnology, Devi Ahilya University, Indore-452001, India \\ *Corresponding author: ak_sbt@yahoo.com
}

\begin{abstract}
The extensive use of petro-based plastics, lack of suitable waste management and casual behaviour of communities to their proper disposal has posed a significant threat to the environment. Currently, single-use petro-based plastics are being banned all over the world due to their inherent non-degradability and problems with disposal. Worldwide, these bans have proved an impetus for increasing research on bio-based polymers as a substitute for conventional plastics. Use of greener or biodegradable polymeric materials would have immense socio-economic and ecological significance.

Polyhydroxyalkanoates (PHAs) are eco-friendly alternatives to petrochemical plastics and are quickly biodegraded in aerobic or anaerobic environments such as a landfill, waste treatment facilities or oceans. PHAs have material and mechanical properties ranging from stiff and brittle crystalline to elastomeric and moulding, similar to petrochemical thermoplastics. In addition, they are hydrophobic, isotactic, biocompatible and have piezoelectric properties. PHAs in being environmentally friendly are seen as ideal candidates for replacing conventional petro-based plastics in numerous fields.

This review focuses on the biochemistry along with in-vitro reaction mechanism of PHA synthases. In addition to the applications and modes of production of PHAs already mentioned a few factors governing PHA synthesis will be discussed. Scope for improving PHA synthesis is also mentioned and some of the tools for enhancing the properties of PHAs are discussed.
\end{abstract}

Keywords: bioplastics; blending; co-culture; PHA; PHA synthase; polyhydroxyalkanoates; recombinant bacteria

\section{Introduction}

Traditional petro-chemical based plastics have been a cause of concern for several decades, not only because of their resistance to biodegradation but also because of their toxic effect on terrestrial and marine environments. Currently, the main source of carbon backbone plastic production is fossil material, which is a non-renewable natural resource and, therefore, of not only environmental concern (Koller 2020). The search for a suitable substitute involved finding new polymers with similar physico-chemical properties and addressing the issue of biodegradation. The first milestone was the discovery by the French microbiologist Maurice Lemoigne in 1920 of a biopolymer, poly (3-hydroxybutyrate), PHB, involved in intracellular granule formation in the gram-positive bacterium, Bacillus megaterium (LeMoigne et al. 1926). This PHB is the most commonly accumulated member of polyhydroxyalkanoates (PHAs) family. PHAs act as microbial storage compounds with versatile plastic like material properties and a possible solution to the above problem.

Although conventional petro-based plastics may not be completely phased out, the increase in socio-consciousness is forcing various industries to change to using polyhydroxyalkanoates (PHAs) polymers for some products because of their eco-friendly properties coupled with thermal, mechanical and physico-chemical properties similar to those of conventional plastics.

They are produced by a wide range of bacteria, which accumulate PHAs intracellularly in granules that act as energy reserves (substitute for fatty acid for maintaining metabolism when under stress) for the microbe. The trigger for this to occur is when a nutrient in a culture medium is limiting but there is an excess of carbon. Under natural conditions, these polymers can constitute nearly $90 \%$ of the dry weight of a cell (Madison and Huisman 1999).

Non-storage PHAs of low molecular weight occur in the cytoplasmic membrane and cytoplasm of Escherichia coli, however.

Chemically, PHAs are polyesters of various hydroxyl alkanoates. More than 150 monomer subunits have been identified in PHAs synthesized by various plants and microbes. The different combinations of these individual subunits offer infinite scope for synthesis of various types of PHAs with different properties. The molecular weight of PHAs differ in different microbes and ranges between 50-1000 KDa. Structurally the individual subunits have the (D) configuration of stereo-specific catalytic enzymes (Senior et al. 1972; Dawes and Senior 1973; Oeding and Schlegel 1973; Wang and Bakken 1998).

An example of a low molecular weight PHA is PHB, which apart from being a green polymer has many of the chemical properties of a conventional plastic like polypropylene (Madison and Huisman 1999).

Microbes are versatile in their biosynthesis of PHAs and use various renewable substances like cellulose, triacyl glycerol, starch and sucrose as precursors. In addition, non-renewable substances such as coal, methane, lignite and mineral oil are also reported as precursors and some microbes also use by-products such as molasses, glycerol and whey, and gases like carbon dioxide as substrates for the synthesis of PHAs. 
Depending on their chemical properties and structure, PHAs may be brittle or rubber-like. These biodegradable polymers are considered to be better than starch-based polymers (starch-polyethylene) and chemically synthesized ones like polylactic acid (PLA) and polyglycolic acid (PGA) due to their structural and tensile properties (Steinbüchel and Fuchtenbusch 1998).

PHAs are biogenic and can be produced naturally in microbial cultures (Steinbüchel and Lutke-Eversloh 2003). These microbial cultures are often referred to as bio-refineries because they are high yielding and tolerant of a wide range of environments. Some algae have also been found to biosynthesize PHAs (Bugnicourt et al. 2014).

The production of biopolymers increased recently because of their sustainability and a desire to increase the use of waste products. This is not only of environmental value, but is also economically beneficial.

\section{Classification of PHAs}

Based on the number of carbon atoms in the monomers and the source of the enzyme, PHAs are classified into two categories: short chain length PHAs (scl-PHAs) and medium chain length PHAs (mcl-PHAs). There are 3 to 5 carbon atoms in the former and 6 to 14 in the latter. Both scl-PHAs and mcl-PHAs are synthesized by different species of bacteria. Examples of bacteria synthesizing scl-PHAs are Cupriavidus necator and Alcaligenes latus, and mcl-PHAs, Pseudomonas putida (Verlinden et al. 2007).

Their properties depend on the number of carbon atoms and their chemical structure. The most studied sclPHA is PHB, which is stiff and brittle, and its crystalline structure makes it difficult to process. Therefore, it is customary to co-polymerize it with poly-3-hydroxyvalerate in order to produce a flexible, strong and thermally stable bio-plastic (Marchessault 1996). The resultant copolymer is 3-hydroxybutyrate-co-3-hydroxyvalerate (PHBV), which is now widely used in the manufacture of items used for the storing and packaging of food. The mclPHAs are used in the production of biomedical products. In contrast to scl-PHAs, mcl-PHAs are more elastic and less crystalline (Gross et al. 1989; Preusting et al. 1990). In most cases, the structural monomeric component(s) of scl-PHAs is 3-hydroxybutyrate (3HB) and of mclPHAs 3-hydroxyoctanoate (3HO) and 3-hydroxydecanoate (3HD) (Anderson and Dawes 1990; Steinbuchel 1991; Steinbuchel and Schlegel 1991; Lee 1996).

\section{Biochemistry of PHA Synthases}

Bacteria use enzymes to biosynthesize PHAs, which remain in the bacteria and act as energy reserves. The rate of biosynthesis and the quantity produced depends on the enzymes involved and physiological status of the bacteria. To understand the complexity of the biochemical processes it is important to know, what enzymes are involved in these pathways.

Four different types of PHA synthases are reported, which differ in their size, number of subunits and substrate specificities. Types I and II specifically synthesize scl-PHAs and mcl-PHAs, respectively, and are composed of a single subunit (PhaC) (Bernd et al. 2003). Types III and IV are dimers composed of the subunits $\mathrm{PhaC}+\mathrm{PhaE}$ and $\mathrm{PhaC}+\mathrm{PhaR}$, respectively. They are implicated in the synthesis of scl-PHAs. The subunits PhaE and PhaR have only an $18 \%$ sequence similarity, but despite this they function similarly in establishing contact between the enzyme and the hydrophobic polymer (Satoh et al. 2002).

Witholt and Kessler (1999) report the presence of an operon in Cupriavidus necator, a Gram negative soil bacterium with three genes, namely $\mathrm{PhaA}, \mathrm{PhaB}$ and $\mathrm{PhaC}$, which code for the enzymes $\beta$-ketothiolase, $(\mathrm{R})$-specific acetoacetyl-CoA reductase and PHA synthase, respectively. These enzymes are involved in the biosynthesis of PHA from acetyl CoA. The first step is the conversion of two molecules of acetyl CoA into acetoacetyl CoA by the enzyme $\beta$-ketothiolase. This reaction requires energy provided by the splitting of ATP into ADP and Pi. In the next step, the reduction of acetoacetyl CoA into 3-hydroxybutyryl CoA with the oxidation of NADPH occurs in the presence of the enzyme (R)-specific acetoacetyl-CoA reductase. Thereafter, 3-hydroxybutyryl CoA is polymerized into polyhydroxybutyrate by PHA synthase.

The mcl-PHAs are generally heteromeric molecules with more than one type of monomeric unit in their structure, whereas mcl-PHAs are usually heteromers of multiple subunits in which the dominant subunit is in direct contact with the carbon chain (Witholt and Kessler 1999). Pseudomonas spp. is the most commonly used for studying mcl-PHA production. The substrates for mcl-PHA are derived from the intermediates produced during biosynthesis and degradation of fatty acids, such as, (R)-3-hydroxyacyl-CoA, which are further polymerized by PhaC. PHA accumulators polymerize hydrophilic monomers into a hydrophobic polymer in the presence of soluble or membrane-bound PhaC (Nobes et al. 2000; Grage et al. 2009). The result is the production of intracellular granules with an amorphous hydrophobic core enclosed in a monolayer of phospholipids embedded in proteins like the PHA synthases, phasins (a regulatory protein) and intracellular depolymerases (Jurasek et al. 2004). The intracellular depolymerases belong to the PhaZ family and are implicated in the remobilization of a carbon supply, whereas phasins are small proteins that regulate the size and morphology of the granules (York et al. 2002).

Schuber et al. (1988) report the expression of recombinant PHA synthase in Escherichia coli and a similar granular morphology to that produced by Pseudomonas. Their genetically engineered Escherichia coli does not have active 
PHA depolymerase and phasins so Gerngross et al. (1994) suggest that there is an indigenous component functionally similar to phasins present in Escherichia coli. When PHA is synthesized in the laboratory (in vitro conditions), the granules of PHA are five times larger than those synthesized by genetically engineered Escherichia coli. It is likely that this because the area available for the naked polymer to coalesce is greater in the absence of a regulatory protein (Gerngross et al. 1994; Nobes et al. 2000).

\section{The in-vitro Reaction Mechanism}

Reaction mechanism, kinetics, substrate specificity and intermolecular interactions are critical parameters in vitro synthesis. Most of the studies on PHA production are on PHA synthases. Type I PHA synthase is the most studied because of its simple structure, though it is possible to purify type II and III enzymes (Jossek et al. 1998; De Roo et al. 2000; Yuan et al. 2001; Takase et al. 2004). The only other highly purified synthase is that of Thermus thermophiles and another from Bacillus cereus SPV, which is in a novel synthase class and class IV synthase, respectively (Lawrence et al. 2005). Purification from the host is a challenge as in this case the synthase is in the form of an aggregate, however, this can be overcome by using recombinant proteins and minute quantities of the detergent Hecameg (Stubbe and Tian 2003).

In-vitro kinetics of type I synthases deviate from the Michaelis-Menten model. The initiation of polymerization is marked by a lag phase, which is followed by an exponential phase. Chromatographic analyses indicate that the synthase remains in an equilibrium between an inactive monomer and active dimer. The in-vivo state still needs to be deciphered (Gerngross et al. 1994).

The lag phase is attributed to the time needed for priming the synthase and polymerization (Stubbe and Tian 2003). Homology studies on the four classes of enzymes indicate a strong similarity with the $\alpha / \beta$-hydrolase superfamily (Bernd 2003). These enzymes have a specific catalytic triad motif with histidine as the nucleophile, which is a suitable catalytic site for interaction between two monomers each contributing cysteine for dimer formation. This may apply to the rest of the three synthase classes and also fits with a polymer being associated with each dimer.

Another model is based on a post-translational modification for fulfilling the need of the second thiol, though this is unlikely for recombinant organisms (Stubbe et al. 2005). The yield of polymers using various classes of synthases indicate that class I and II synthases produce a single polymer chain per molecule of enzyme while class III synthases result in chain transfer reaction, which yield several polymer chains per enzyme molecule (Jossek et al. 1998). Nonetheless, in vivo studies class I synthases can yield 60 polymer chains (Tian et al. 2005).

It is suggested that the termination of polymerization is brought about by the attachment of a nucleophilic chain transfer molecule at or near the active site (Lawrence et al. 2005). It is predicted that CoA (Kurja et al. 1995; Jossek et al. 1998), hydroxybutyrate (Madden et al. 1999), water (Kawaguchi and Doi 1992; Kusaka et al. 1997) or alcohol (Shi et al. 1996; Madden et al. 1999) may act as nucleophiles. The difference in the yield in-vivo and in-vitro conditions may be attributed to either the lack of phasin-like regulatory proteins in-vitro or high concentration of nucleophilic agent in-vivo. This would account for the high molecular mass of in-vitro synthesized PHAs as the scope there for chain transfer is greatly reduced (Daae et al. 1999; Lawrence et al. 2005).

The study of the biosynthesis and production of PHA is mainly dependent on $R$. eutropha. Two acetyl-CoA moieties are condensed to form acetoacetyl-CoA by $\beta$-ketothiolase (PhaA) in R. eutropha, which then undergoes reduction by NADPH-dependent reductase (PhaB) resulting in the formation of the (R)-isomer of 3-hydroxybutyryl-CoA.

Similar PHA biosynthesis is also reported in $R$. rubrum in which the reductase is an NADH-dependent isoenzyme that produces (S)-isomers of 3-hydroxybutyryl-CoA. Here two groups of enoyl-CoA hydratases facilitate the conversion of ( $\mathrm{S}$ )-type to (R)-type isomer, which is the only form of stereoisomer that is accepted by the polymerizing enzyme, PHA synthase. Studies on the biosynthesis of PHA in R. eutropa and R. rubrum have revealed a cascade of events that result in the efficient production of PHA in-vivo (Sudesh et al. 2000).

The biodegradability of the PHAs is another aspect that needs to be considered. PHAs act as energy resource for microbes, which enzymatically hydrolyse them into $\mathrm{HB}$ and HV units. The degraded products further facilitate biomass growth in culture. The rate of degradation is dependent on parameters like exposed surface area, $\mathrm{pH}$, temperature, humidity and other ingredients in the system. $\mathrm{P}(\mathrm{HB}-\mathrm{HV})$ remains stable in air for an indefinite period and is therefore resistant to degradation under ambient conditions. Under aerobic conditions, PHA degrades into water and carbon dioxide. The rate of degradation is fastest in oxygen-depleted sewage and slowest in marine environments (Doi et al. 1992; Lee et al. 1996).

\section{Applications}

The PHA family of products have numerous uses, especially for making containers for food since these polymers are inert and non-toxic. In addition, because of their anti-microbial properties, they are used for manufacturing adhesive films and hygiene-related baby items, including nappies. They can also be used in printing as toners and adhesives for coating films (Madison and $\mathrm{Hu}$ isman 1999).

Polyhydroxyalkanoates (PHAs) that accumulate as natural biogenic polyesters in microbial cultures, when formulated and processed, form highly flexible materi- 


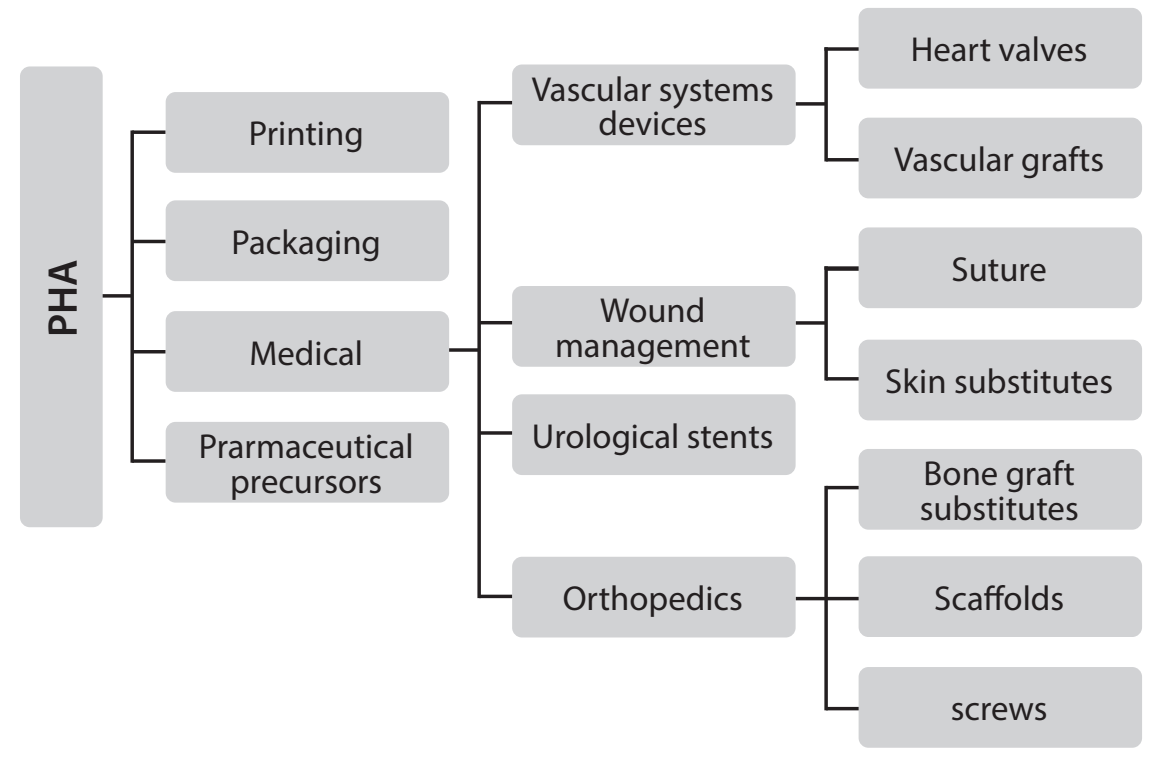

Fig. 1 A flow chart indicating the various uses of PHAs.

als that are sustainable substitutes for traditional plastics. As bio-plastics, they are used in the manufacture of electronic products like mobile phones and computer tablets etc. Their amphiphilic property makes them suitable for encapsulating seeds and fertilizers (causing slow release), biodegradable films and containers for covering and storage of crops. In addition, the turnover in agriculture is likely to increase due to the use of their waste materials for the production of PHAs.

PHAs also have many medical uses because their biodegradable and non-toxic properties make them suitable for manufacturing biomaterial for insertion in the human body. They are used as surgical sutures, adhesive bandages, orthopaedic straps and pins, repair stents, in targeted drug delivery, patches and matrix for bone marrow. When combined with hydroxyapatite it becomes a bioactive composite suitable for bone tissue regeneration and replacement (Chen and Wu 2005). There are many possible commercial uses of PHAs derived bio-plastics. A number of the applications in which bioplastics are being used are depicted in Fig. 1.

\section{Production of PHAs}

Biosynthetic polymers can be produced by plants or microbes. The mass production of bio-plastics with different properties using microbes is costly, requires skill, technical expertise and the yield is low compared to petro-chemical based plastics, which has inhibited the industrial use of PHAs (Ojumu et al. 2004).

\section{Natural Biosynthesis of PHAs}

Various factors determine the subunit composition of a polymer, such as, type of microbe, composition of me- dia, fermentation and mode of recovery. There are more than 300 species of Gram positive and Gram-negative microbes capable of PHA biosynthesis (Steinbüchel and Schlegel 1991; Suriyamongkol et al. 2007). These microbes have the necessary enzymes for the biosynthesis of PHAs and store them as granules in their cytoplasm as an energy reserve (Reddy et al. 2003). The stimulus for their biosynthesis is usually some kind of stress like lack of a nutrient ( $\mathrm{N}$ or $\mathrm{P}$ ) in the presence of excess carbon (Dawes and Senior 1973; Amass et al. 1998). However, a few species like Azotobacter vinelandii (mutant and wild type), A. eutrophus and A. latus also synthesize PHAs under nonstress conditions (Ojumu et al. 2004). Studies indicate that the most common limitation in almost all types of bacteria to the accumulation of PHA, is the availability of nitrogen. However, in some species of bacteria, such as Azotobacter, it is the availability of oxygen (Johnston et al. 2017).

\section{PHAs Production by Recombinant Bacteria}

As it has been mentioned above, there are number of microbes, which synthesize PHAs as an energy reserve, when either stressed or routinely. These organisms, however, grow slowly and have a long generation time. The extraction of PHAs from these microbes is done using lysis, which results in a very low yield. It also depends on the physiological conditions of the cells since they also have enzymes, which degrade these PHAs, when they require the energy.

The wild type E. coli lack PHAs otherwise its ability to grow rapidly make it a good potential candidate for the production of PHAs. If E. coli can be genetically engineered to produce enzymes for synthesizing PHAs, then the production and isolation of PHAs is cost-effective (Steinbeuchel and Schlegel 1991; Madison and Huisman 1999; Kadouri et al. 2005). 
There have been attempts to characterize the metabolic pathways, so that it is possible to insert various substances at different stages. Recombinant strains of $E$. coli that have genes for the biosynthesis of PHA from Alcaligenes eutrophus have been produced by metabolic engineering, which yield high levels of PHA (Zhang et al. 1994; Lee et al. 1996). E. coli is highly versatile in terms of the range of substances it can utilize from lactose, glucose and sucrose to xylose and more complex sugars, which further increases the cost-effectiveness of the process. Cheaper substrates like whey and hemicellulose hydrolyzates can also be used (Lee et al. 1996).

\section{Production of PHA by Genetically Engineered Plants}

Not only microbes but plants also produce PHA, with crop plants high on the list, which are even more cost-effective than bacteria or yeast in terms of yield and effort. The production of PHA using crops requires the genetic engineering of transgenic varieties capable of high PHA production and remaining genetically stable for many generations (Snell and Peoples 2002). This would enable the production of these green polymers in greater quantities than other ways of producing PHA. Unlike bacterial cells, plant cells are compartmentalized and transformed phb genes allocated to locations with high concentrations of acetyl-CoA. Arabidopsis thaliana is the favoured choice for such transgenic transformation, as like E. coli, it is the equivalent in terms of plants (Madison and $\mathrm{Hu}$ isman 1999). The innate presence of 3-ketoacyl-CoA thiolase in $A$. thaliana required the transfection with phbB and phbC genes from $R$. eutropha for it to accumulate PHB in the nucleus, cytoplasm and vacuoles of its cells; however the plant has several growth defects. This was resolved by targeting the phbCAB genes in the plastid of $A$. thaliana, which receive a high flux of carbon via acetyl CoA, which enhance the yield of PHA without adversely affecting the growth of the plant. The maximum yield in terms of dry weight percentage is 14\% (Nawrath et al. 1994). Oilseed plants are also potential candidates for the production of seed specific PHAs. The common source of PHB and oil is acetyl-CoA, which is easily inserted into the biosynthesis pathway of PHA with a high innate carbon flux courtesy of acetyl CoA. Other plants being used in PHA production are Gossypium hirsutum and Zea mays.

\section{Co-culturing and the Biosynthesis of PHA}

Co-culturing using two or more microorganisms to produce PHA from complex carbon feed (Choi and Lee 1999; Ganduri et al. 2005; Nikodinovic-Runic et al. 2009) is easy as several microbes are available. The synergistic production of PHA in such cultures is attributed to the specific utilization of the same substrate by different bac- terial strains. The most well-known example is the utilization of toxic compounds like benzene, toluene, ethyl acetate, xylene (collectively called BTEX) and styrene in the synthetic plastic pyrolysis of oil for conversion to biodegradable PHA by three strains of Pseudomonas putida, namely F1, mt-2 and CA-3.

\section{Factors Governing PHA Synthesis}

\section{Species of bacteria}

A major factor determining the efficiency of PHA synthesis is a species of microbe that is high yielding when provided with a cheap substrate. Industrial application requires mass scale production for which rapidly growing species and strains are required, such as recombinant organisms with short generation times and are easy to lyse. A good example is recombinant E. coli, which is used to produce $\mathrm{PHB}$ along with other co-polymers (Schubert et al. 1988; Slater et al. 1988; Zhang et al. 1994; Reddy et al. 2003). Several strains of Pseudomonas, like P. oleovorans, have been engineered to produce various blends of the polymers scl- and mcl-PHA (Preusting et al. 1993).

\section{Culture medium}

A culture medium, apart from providing conditions for optimal growth of the microorganisms, also determines the yield and cost of the product, as it is the major cost of producing PHA on an industrial scale. Therefore, there is a need to reduce the cost of making the medium without compromising on essential ingredients needed for high productivity (Lee 1996; Ojumu et al. 2004), which are corn steep liquor (Nikel et al. 2006), molasses (Solaiman et al. 2006), rice bran and wheat (Huang et al. 2006; Van-Thuoc et al. 2008), starch-rich waste (Kim and Chang 1998), activated sludge (Yuan et al. 2006; Jiang et al. 2009), effluents from olive and palm oil mills (Ribera et al. 2001; Pozo et al. 2002; Bhubalan et al. 2008) and whey (Marangoni et al. 2002; Koller et al. 2008). The medium selected depends on the culture organism. In addition, by varying the medium used to produce homopolymers or heteropolymers it is possible to produce more than 100 different monomers, which can be combined to produce homopolymers or copolymers with molecular weights ranging between 50 to $100 \mathrm{KDa}$ (Taguchi and Doi 2004; Valappil et al. 2007).

\section{Fermentation conditions}

This process involves first achieving a high density of microbes and then creating a nutrient-limited condition that enhances the synthesis of PHA (Asenjo et al. 1995; Madison and Huisman 1999; Tsuge 2002). The temperature and $\mathrm{pH}$ of the medium are determined by the species of microorganism used. The temperature is kept at $30-37^{\circ} \mathrm{C}$ and slow stirring determines its oxygen concentration, while the $\mathrm{pH}$ is varied depending on substrate (Kim et al. 1994; Chung et al. 1997). Various 
things can be done to produce fermentation conditions suitable for maximum yield, such as varying the source of carbon during the process (Kim et al. 1992; Koyama and Doi 1995; Henderson and Jones 1997; Durner et al. 2001; Zinn et al. 2003), diluting the medium (Sun et al. 2007), fed and unfed culture batches (Kim et al. 1992; Rhee et al. 1993; Kim and Chang 1995; Kim et al. 1997) and limiting the supply of nitrogen, phosphorus or oxygen (Sun et al. 2007).

\section{Recovery}

The final step in the biosynthesis is recovery the cost of which is dependent on the method of purification. The most primitive of which is extraction using an organic solvent (Lee 1996), which is better in terms of the percentage recovery of PHA than cell lysis followed by aqueous extraction, since cell lysis alters the molecular mass of the polymer (Ojumu et al. 2004). Other methods include enzymatic digestion of non-PHA components, floatation-mediated extraction using supercritical carbon dioxide and spontaneous liberation of polymers. Because of their limitations, however, neither are suitable for industrial use.

\section{Methods for Improving the Properties of PHAs}

\section{Blending}

Blending of PHAs results in an appreciable improvement in the properties of the biopolymers. The raw materials determine the properties of the final product in terms of its physical and mechanical durability, whereas by varying their concentrations and optimizing the temperature and $\mathrm{pH}$ alter the biodegradability and toxicity of the bio-plastics. Currently, this is very important due to increase in societal and government concerns over non-sustainable energy resources. The blending has also proved to be a boon for the medical industry, which is using this method in the manufacture of implants and absorbable non-toxic surgical sutures.

\section{Components Used in Blending}

\section{Natural raw material}

Starch is the most widely used as it is highly biodegradable, readily available and convenient (Pilkington et al. 2015). The linear amylose and branched amylopectin structure of starch determines the mechanical properties of the final product. It is reported that it results in a significant improvement in the properties of $\mathrm{PHB}$ and a reduction in the cost of production (Godbole et al. 2003). PHB and all percentages of it in blends of starch have the same transition temperature $(\mathrm{Tg})$ and the final product in all cases is crystalline. The tensile strength of the product is best when the percentage of starch is $30 \%$ (Pilkington et al. 2015), which is what the packaging industry requires for paper and cardboards packaging etc. The amends were also possible with the thermal and phase separation properties by starch blending. The final blend is immiscible as there is a direct relationship between $\mathrm{Tm}$ and percentage of starch (Zhang et al. 1997a). The enthalpy of melting did not change significantly while $\mathrm{Tg}$ of $9^{\circ} \mathrm{C}$ is required for all percentages of starch.

However, in spite of reducing manufacturing costs and improvements in mechanical properties, the final products are brittle and unsuitable for producing strong biofilms, but this can be overcome by using copolymer grafts of starch and glycidyl methacrylate (GM), which increase the compatibility between the bio-films and PHA matrix (Willett et al. 1998). Treatment of starch with GM results in the polymer being more durable and tensile and there is a direct correlation between this property and percentage of treated starch. Scanning electron microscopy revealed strong adhesion between the film and matrix. By using corn starch treated with polyvinyl acetate (PVAc) also increases the mechanical strength of the film (Lai et al. 2015). When the percentage is $27 \%$ the $\mathrm{Tg}$ increases to $44^{\circ} \mathrm{C}$ and the compatibility with PHB is maintained. This blend is also compatible with the recovery method as it does not affect the $\alpha$-amylase degradation of corn starch (Lai et al. 2015).

\section{Cellulose derivatives}

Cellulose derivatives like cellulose acetate, cellulose butyrate, cellulose propionate or ethyl cellulose are potential candidates for PHB blends, especially for materials used in the biomedical industry (Zhang et al. 1997b). They are compatible with PHAs and are easily recoverable (Wang et al. 2015). Studies on crystallization, melting properties and miscibility of these blends indicate a concentration dependent increase in $\mathrm{Tg}$ with decrease in the percentage of PHB. These blends lack crystalline properties in non-isothermal crystallization tests, however, spherulite formation continues but is delayed (El-Shafee et al. 2001). The single Tg indicates the blends are miscible and the phase morphology analysis that the polymer is homogeneously amorphous. The presence of cellulose acetate butyrate in the inter-lamellar region of PHB enhances the mechanical properties of the final blend.

\section{Lignin}

The functional groups of lignin (phenyl propane repeats and carboxylic acid groups) determine its amorphous nature, which in turn make it a suitable candidate for producing PHA blends. The phase morphology of lignin prevents crystal nucleation and spherulite formation, and decreases the brittleness reported when using cellulose derivatives. Lignin and its derivatives are highly miscible with PHB and prevent crystal formation and reduce the number and size of spherulites (Weihua et al. 2004; Mousavioun et al. 2013). Soda lignin is another modified derivative that has a significant effect on the thermal stability of PHB (Mousavioun et al. 2010). Ther- 
mo-gravimetry and electron microscopy indicate that the optimal percentage of soda is $40 \%$. The intermolecular interactions between the blending components are due to hydrogen bond formation between carbonyl groups of PHB and carboxyl groups of lignin. This is the major reason for the enhanced fractural stability of this blend and also prevents degradation (Mousavioun et al. 2012). During degradation, there is an increase in the unevenness of the surface of the bio-film, which indicates efficient biodegradation.

\section{Co-polymerization}

\section{Different monomeric subunits of PHA}

Production of copolymers using different combinations of monomers is a common commercial practice. The different monomers are important in determining the strength and durability of the biopolymer. PHB is brittle and tends to form spherulites. Blending PHB with PHA-mcl monomers imparts thermal and mechanical properties similar to polyolefin (Saad 2002). These combinations are also an effective way of producing polyester-like blends and products for biological applications. Different blends of poly-3-hydroxybutyrate (P3HB) and poly-4-hydroxybutyrate ( $\mathrm{P} 4 \mathrm{HB})$ vary from crystalline to elastic and the use of different blends of PHBHHx with $\mathrm{PHB}$ have resulted in the production a wide range of biomedical products (Yoshie et al. 2004) and the modification of the properties of biological materials. PHB rapidly crystallizes and forms an inundated bio-film that is not a suitable matrix for mammalian cells, but by incorporating copolymers into blends alters the degree of crystallisation and results in an even surface for attachment and growth of cells (Kai et al. 2003).

\section{Synthetic Biodegradable Polymers}

\section{Poly-lactic acid (PLA)}

Poly-lactic acid is produced from lactic acid or by fermenting simple carbohydrates. It is biodegradable and therefore is widely used to produce biodegradable packaging and biocompatible devices. Despite being an environmentally friendly option, its poor durability and thermal stability hinders its use in the industrial production of polymers. Blending mineral fillers with PHA/PLA results in immiscible polymers with a nodular morphology (Koyama and Doi 1997; Ohkoshi et al. 2000; Gerard and Budtova 2012) and those with PHBV enhancing the thermal stability of PLA. Despite being brittle, these bio-films are very ductile and plastic (Gerard and Budtova 2012). The ductile strength can be increased by including more PHA-mcl than PHA-scl co-polymers in the blend., which resulted in Noda et al. (2004) hypothesizing that PLA blends with PHA can compensate for the limitations of the individual ingredients in a combination (Noda et al.
2004). An addition of $10 \%$ PHA to the blend transforms the phase morphology from crystalline to amorphous and PHA mcls and epoxy-PHA mcls results in amorphous elastomers. This is attributed to the interaction of the epoxy group with the hydroxyl in the PLA (Takagi et al. 2004). The method of blending also affects the miscibility and compatibility imparted to the final product. PHA/PLA blends prepared using solvent casting and melt blending methods vary in their miscibility (Zhang et al. 1996), with the former immiscible for a range of percentages and the latter appreciably miscible, which affect the crystalline properties of the product (Furukawa et al. 2007). Those PLA blends with low crystallization temperatures are miscible but those with high crystallization temperatures are not.

\section{Polycaprolactone (PCL)}

Because of its mechanical and ductile strength, it is extensively used in medical applications, which have undergone several modifications. It is inherently semi crystalline and blending with PHBHHx yields a soft and flexible polymer suitable for musculoskeletal tissue engineering (Lim et al. 2013). The biodegradable and non-toxic nature of this blend makes it even more suitable for tissue engineering, adhesion and proliferation surfaces for human foetal mesenchymal tissue and human bone (Chen and $\mathrm{Wu} 2005)$. The ductility of PCL/PHBV blends is directly proportional to the percentage of PCL in the blend (Chiono et al. 2008). Even vascular grafts are prepared using these blends, which are biocompatible with the rat cerebral endothelial cells in terms of adhesion, proliferation, viability and migration over a period of two weeks (Del Gaudio et al. 2012).

\section{Chemical Modification of PHAs}

This is another tool for enhancing the properties of PHAs, which is more regulated than blending as it modulates the structure of the polymer and the resulting changes are mostly predictable. This can be taken to the next level by controlled polymerization, which impart a new dimension to the architecture of the PHA. PHA graft graphene nano-composites degrade at higher temperatures and have a higher electrical conductivity than neat PHA (Yao et al. 2019).

\section{Grafting on natural polymers}

Grafting PHA onto natural polymers have proved highly suitable for medical applications including tissue engineering, production of antimicrobial material and drug delivery. The interaction of the $-\mathrm{NH} 2$ group of chitosan with $-\mathrm{COOH}$ group of $\mathrm{PHB}$ and the ester formation between $-\mathrm{COOH}$ of $\mathrm{PHB}$ and $-\mathrm{OH}$ of cellulose produces two variants of $\mathrm{PHB}$ graft copolymers (Yalpani et al. 1991). Hydrophilic nature of chitosan imparts an amphiphilic tendency to the graft copolymer and there is 
a direct correlation between the solubility of the final biopolymer and the degree of the graft (Arslan et al. 2007).

\section{PHA graft copolymers with vinyl or (meth)acrylate groups}

Copolymerization of PHA grafts with oligomers containing vinyl or (meth)acrylate groups like 2-hydroxyethylmethacrylate, polyethylene glycol or poly(methylmethacrylate) by irradiation is highly effective in altering the property of PHA grafts (Hazer and Steinbüchel 2007). The presence of vinyl chains enhances the hydrophilicity of the polymer, which reduces the inter-facial tension and platelet adhering property of the product, hence making it suitable for the manufacture of blood containing bags and devices (Chung et al. 2003).

\section{Scope for Improving PHA Production}

With increased demand for environmentally friendly PHA there is a need to consider the sustainability and cost-effectiveness of its synthesis.

\section{Screening of New Species of Microbes}

There is a need to explore a wide range of habitats for more microorganisms for PHA production, especially those in estuaries, rhizospheres and wastewater effluents, and develop measures for distinguishing between efficient and non-efficient PHA producers. Genetic interventions like using recombinant organisms with suitable enzyme requirements and the identification of PHA producing species using FISH or PCR has facilitated this process. Mixed microbial cultures (MMC) are being widely adopted in preference to recombination as MMC creates an ecosystem with various species contributing to the biosynthesis of PHA. The medium for the microbes and synthesis conditions are provided by bioreactors (Duque et al. 2014). Use of agro-industrial waste as the raw material in these bioreactors for producing PHA has proved very successful. Similarly, the fermentation of agro-waste like sugar cane molasses, olive oil effluents and even food waste in MMC systems (Koller et al. 2017). The production of PHA in photo bioreactors with mixotrophic microbe culture containing cyanobacteria (recombinant or wild type) is sustainable and economic (Stal 1992; Narodoslawsky et al. 2015), with the process optimal in terms of carbon dioxide emission and soluble organic compounds in cultures with Nostoc muscorum Agardh acting on poultry waste (Bhati and Mallick 2016).

\section{Genetic interventions for improvement of PHA producers}

Molecular biology has provided tools that can be used to convert non-PHA synthesisers to producers or for improving yield and properties of PHA. Recombinant E. coli cloned with PHAc synthases on a lactose substrate produces PHB (Ahn et al. 2001). In another study, Cupriavi- dus necator was engineered to exploit more substrates in a medium and produce more PHB. The lac system genes (lac0, lacZ and lacI) can be integrated into the bacterial genome for allowing the conversion of lactose to polyesters (Povolo et al. 2010). In addition, genetic tools have facilitated improvements in the recovery process, for example, PHAmcl can be produced in vivo if staphylococcus nuclease is integrated into the genome of Pseudomonas putida. Currently this is the most effective way for producing medium chain length PHA (Boynton et al. 1999). P. putida with knock-outs of fatty acid degradation enzymes and the substrate carrier protein, 3-hydroxyacyl-CoA-acyl, is able to produce homomers instead copolymers due to reduction of $\beta$-oxidation by these mutants (Liu et al. 2011).

\section{Utilization of inexpensive agro-waste as raw material}

Fifty percent of the cost of producing PHA is the cost of the substrate and subsequent processing. The most common raw materials are simple sugars, fatty acids and oils, however, there are alternative carbon-rich resources. The waste from agriculture is a rich source of carbon that can be used as a substrate for the synthesis of PHA. Agro-waste is a by-product of a variety of crops, such as, the bagasse from fruit and sugarcane, peel from potato, orange and banana, and bran of wheat and rice (Stavroula et al. 2020). The geographical availability is restricted to tropical countries, like India. It is estimated that for every 10 tonnes of sugarcane, 3 tonnes of bagasse is produced (Kulkarni et al. 2014). Further, as India is the world's largest producer of bananas there is a great potential for it to use the peel to produce PHA. Sugarcane and corn cob are the richest sources of carbon used in media for culturing C. necator. When considering a substrate for synthesis of PHA it is important to determine whether it is readily available all year round, cheap and easy to store in good condition for long periods. The agricultural substrates are first converted to stable sugars like lactose via anaerobic fermentation and then aerobically used to produce PHA. In addition, green gas effluents from anaerobically produced silage can be added to $C$. necator cultures producing PHA (Koller et al. 2005). The major challenge in using agro- or industrial waste as substrates is the poor yield of PHAs. Purified sugars deliver higher yields as they provide higher concentrations of the substrate for synthases to act on, whereas agro- or industrial waste, not only is the concentration of the carbon source less but there are other ingredients inhibiting or slowing down the synthesis. The dilution of the substrate increases the production time, which increases the operation costs, especially, in mass scale production. There is, therefore, a need to look for ingredients rich in carbon that optimise the process and reduce the cost. Lactose-rich whey protein consists of about $4-5 \%$ carbon by weight, but after ultrafiltration, one is left with a lactose-rich feedstock (Ahn et al. 2001). Whey protein can also be enzymatically broken down into glucose and galactose and after dehydration the mix consists of $50 \%$ sugars by weight. Charcoal stripping wood is good 
for removing inhibitors in the reaction mix (Koller et al. 2005). Liquefied wood is produced from spruce wood dust via a microwaved toluene sulfonic acid mediated hydrolysis. Although it is a good substrate for PHA production by $C$. necator, it is also contains an abundance of inhibitors, which are removed cheaply by means of charcoal media filtration in order to restore the growth kinetics of the reaction. The use of liquefaction is widely criticised because it introduces toxins and inhibitory substances into the process. The extreme temperatures, pressures and $\mathrm{pH}$ leads to the production of inhibitors like furfural and 5-hydroxymethyl furfural. This can be avoided by using enzymatic hydrolysis under normal conditions, however, the many hydrolytic enzymes required make it very expensive (Koller et al. 2017). In the circumstances charcoal stripping is an effective way of producing a substrate (bagasse hydrolysate) suitable for Burkholderia cepacia and B. sacchari (Silva et al. 2004). As these two Burkholderia strains are able to utilise both pentose and hexose sugars in the bagasse hydrolysate, they give higher yields than those obtained using a purified sugar substrate (xylose). Charcoal stripping is also used for removing inhibitors from chicory root hydrolysate for fermentation by $C$. necator (Haas et al. 2015). An economic alternative to charcoal bed filtration for removing inhibitory components like free fatty acids, ash, residual biodiesel and methanol is demethanolization, which involves thermal or vacuum - assisted evaporation (Hájek et al. 2012). Phase separation techniques or even distillation and vacuum dehydration are useful for concentrating glycerol. Consequently, a multi-culture system can use both glycerol and methanol as substrates. Methylomonas extorquens has been used in such studies, in which a crude glycerol medium resulted the diauxic growth of the microbe and conversion of methanol before the utilisation of glycerol (Braunegg et al. 1999). Using crude glycerol as the sole carbon source results in a high yield of PHB in sequencing bioreactors (Moita et al. 2014). PHA production is also associated with the generation of biofuel. Integration of these two industries that have similar feed stocks would be a step closer to sustainability.

\section{Conclusion}

PHAs are extremely important because they enable society to decouple economic growth from resource exhaustion and environmental ruin. With increase in the diversity of PHAs there will be a considerable increase in their application. However, the cost of production of PHAs constrains their large-scale industrialization and commercialization. A lower-cost can be achieved by integrating fields like synthetic biology, systems biology, morphology engineering, next generation industry biotechnology and high-cell-density cultivation, which will facilitate the synthesis of all kinds of PHA. Moreover, bacteria can be designed that have multiple metabolic pathways and can synthesize a particular PHA on demand. Eventually, an economically, ethically and environmentally sustainable production of PHA is feasible.

\section{Acknowledgements}

SS acknowledges the award of a Golden Jubilee Fellowship from Devi Ahilya University, Indore. Authors acknowledge the use of facilities of the Department of Biotechnology, Ministry of Science and Technology, Government of India, New Delhi (DBT) present in the Bioinformatics Sub Centre and M.Sc. Biotechnology program.

\section{REFERENCES}

Ahn WS, Park SJ, Lee SY (2001) Production of poly (3-hydroxybutyrate) from whey by cell recycle fed-batch culture of recombinant Escherichia coli. Biotechnol Lett 23: 235-240.

Amass W, Amass A, Tighe B (1998) A review of biodegradable polymers: uses current developments in the synthesis and characterization of biodegradable polyesters blends of biodegradable polymers and recent advances in biodegradation studies. Polym Int 47: 89-144.

Anderson AJ, Dawes EA (1990) Occurrence, metabolism, metabolic role and industrial uses of bacteria polyhydroxyalkanoates. Microbiol Rev 54: 450-472.

Arslan H, Hazer B, Yoon SC (2007) Grafting of poly (3-hydroxyalkanoate) and linoleic acid onto chitosan. J Appl Polym Sci 103: 81-89.

Asenjo JA, Schmidt AS, Andersen PR, Andrews BA (1995) Effect of single nutrient limitation of poly- $\beta$-hydroxybutyrate molecular weight distribution in Alcaligens eutrophus. Biotechnol Bioeng 46: 497-502.

Bernd HA (2003) Polyester synthases: natural catalysts for plastics. Biochem J 376: 15-33.

Bhati R, Mallick N (2016) Carbon dioxide and poultry waste utilization for production of polyhydroxyalkanoate biopolymers by Nostoc muscorum Agardh: a sustainable approach. J Appl Phycol 28: 161-168.

Bhubalan K, Lee WH, Loo CY, Yamamoto T, Tsuge T, Doi Y, Sudesh K (2008) Controlled biosynthesis and characterization of poly (3-hydroxybutyrate-co-3-hydroxyvalerate-co-3-hydroxyhexanoate) from mixtures of palm kernel oil and $3 \mathrm{HV}$-precursors. Polym Degrad Stabil 93: 17-23.

Boynton ZL, Koon JJ, Brennan EM, Clouart JD, Horowitz DM, Gerngross TU, Huisman GW (1999) Reduction of cell lysate viscosity during processing of poly (3-hydroxyalkanoates) by chromosomal integration of the staphylococcal nuclease gene in Pseudomonas putida. Appl Environ Microb 65: 1524-1529.

Braunegg G, Genser K, Bona R, Haage G, Schellauf F, Winkler E (1999) Production of PHAs from agricultural waste material. Macromol Sy 144: 375-383.

Bugnicourt E, Cinelli P, Lazzeri A, Alvarez VA (2014) Polyhydroxyalkanoate (PHA): Review of synthesis characteristics processing and potential applications in packaging. Mater Sci.

Chen GQ, Wu Q (2005) Microbial production and applications of chiral hydroxyalkanoates. Appl Microbiol Biot 67: 592-599.

Chiono V, Ciardelli G, Vozzi G, Sotgiu MG, Vinci B, Domenici C, Giusti P (2008) Poly (3-hydroxybutyrate-co-3-hydroxyvalerate)/poly (?-caprolactone) blends for tissue engineering appli- 
cations in the form of hollow fibers. J Biomed Mater Res A 85: 938-953.

Choi J, Lee SY (1999) Factors affecting the economics of polyhydroxyalkanoate production by bacterial fermentation. Appl Microbiol Biot 51: 13-21.

Chung CW, Kim HW, Kim YB, Rhee YH (2003) Poly (ethylene glycol)-grafted poly (3-hydroxyundecenoate) networks for enhanced blood compatibility. Int J Biol Macromol 32: 17-22.

Chung YJ, Cha HJ, Yeo JS, Yoo YJ (1997) Production of poly (3-hydroxybutyric-co-3-hydroxyvaleric) acid using propionic acid by $\mathrm{pH}$ regulation. J Ferment Bioeng 83: 492-495.

Daae EB, Dunnill P, Mitsky TA, Padgette SR, Taylor NB, Valentin HE, Gruys KJ (1999) Metabolic modeling as a tool for evaluating polyhydroxyalkanoate copolymer production in plants. Metab Eng 1: 243-254.

Dawes EA, Senior PJ (1973) The role and regulation of energy reserve polymers in micro-organisms. In: Advances in microbial physiology, Academic Press 10, pp 135-266.

De Roo G, Ren Q, Witholt B, Kessler B (2000) Development of an improved in vitro activity assay for medium chain length PHA polymerases based on coenzymeA release measurements. J Microbiol Methods 41: 1-8.

Del Gaudio C, Fioravanzo L, Folin M, Marchi F, Ercolani E, Bianco A (2012) Electrospun tubular scaffolds: on the effectiveness of blending poly ( $\varepsilon$-caprolactone) with poly (3-hydroxybutyrate-co-3-hydroxyvalerate). J Biomed Mater Res B 100: $1883-1898$.

Doi Y, Kawaguchi Y, Koyama N, Nakamura S, Hiramitsu M, Yoshida Y, Kimura U (1992) Synthesis and degradation of polyhydroxyalkanoates in Alcaligenes eutrophus. FEMS Microbiol Rev 103: $103-108$

Duque AF, Oliveira CS, Carmo IT, Gouveia AR, Pardelha F, Ramos AM, Reis MA (2014) Response of a three-stage process for PHA production by mixed microbial cultures to feedstock shift: impact on polymer composition. New Biotechnol 31: 276-288.

Durner R, Zinn M, Witholt B, Egli T (2001) Accumulation of poly [(R)-3-hydroxyalkanoates] in Pseudomonas oleovorans during growth in batch and chemostat culture with different carbon sources. Biotechnol Bioeng 72: 278-288.

El-Shafee E, Saad GR, Fahmy SM (2001) Miscibility crystallization and phase structure of poly (3-hydroxybutyrate)/cellulose acetate butyrate blends. Eur Polym J 37: 2091-2104.

Furukawa T, Sato H, Murakami R, Zhang J, Noda I, Ochiai S, Ozaki Y (2007) Comparison of miscibility and structure of poly (3-hydroxybutyrate-co-3-hydroxyhexanoate)/poly (1-lactic acid) blends with those of poly (3-hydroxybutyrate)/poly (l-lactic acid) blends studied by wide angle X-ray diffraction differential scanning calorimetry and FTIR microspectroscopy. Polymer 48: 1749-1755.

Ganduri VSRK, Ghosh S, Patnaik PR (2005) Mixing control as a device to increase $\mathrm{PHB}$ production in batch fermentations with co-cultures of Lactobacillus delbrueckii and Ralstonia eutropha. Process Biochem 40: 257-264.

Gerard T, Budtova T (2012) Morphology and molten-state rheology of polylactide and polyhydroxyalkanoate blends. Eur Polym J 48: 1110-1117.

Gerngross TU, Snell KD, Peoples OP, Sinskey AJ, Csuhai E, Masamune S, Stubbe J (1994) Overexpression and purification of the soluble polyhydroxyalkanoate synthase from Alcaligenes eutrophus: evidence for a required posttranslational modification for catalytic activity. Biochemistry 33: 9311-9320.

Godbole S, Gote S, Latkar M, Chakrabarti T (2003) Preparation and characterization of biodegradable poly-3-hydroxybutyrate-starch blend films. Bioresour Technol 86: 33-37.
Grage K, Jahns AC, Parlane N, Palanisamy R, Rasiah IA, Atwood JA, Rehm BH (2009) Bacterial polyhydroxyalkanoate granules: Biogenesis structure and potential use as nano-/micro-beads in biotechnological and biomedical applications. Biomacromolecules 10: 660-669.

Gross RA, DeMello C, Lenz RW, Brandl H, Fuller RC (1989) Biosynthesis and characterization of poly(B-hydroxyalkanoates) produced by Pseudomonas oleovorans. Macromolecules 22: $1106-1115$

Haas C, Steinwandter V, Diaz De Apodaca E, Maestro Madurga B, Smerilli M, Dietrich T, Neureiter M (2015) Production of PHB from chicory roots-comparison of three Cupriavidus necator strains. Chem Biochem Eng Q 29: 99-112.

Hájek M, Skopal F, Čapek L, Černoch M, Kutálek P (2012) Ethanolysis of rapeseed oil by $\mathrm{KOH}$ as homogeneous and as heterogeneous catalyst supported on alumina and $\mathrm{CaO}$. Energy 48: 392-397.

Hazer B, Steinbüchel A (2007) Increased diversification of polyhydroxyalkanoates by modification reactions for industrial and medical applications. Appl Microbiol Biot 74: 1-12.

Henderson RA, Jones CW (1997) Physiology of poly-3-hydroxybutyrate (PHB) production by Alcaligenes eutrophus growing in continuous culture. Microbiology 143: 2361-2371.

Huang TY, Duan KJ, Huang SY, Chen CW (2006) Production of polyhydroxyalkanoates from inexpensive extruded rice bran and starch by Haloferax mediterranei. J Ind Microbiol Biot 33: 701-706.

Jiang Y, Chen Y, Zheng X (2009) Effecient polyhydroxyalkanoates production from a waste-activated sludge alkaline fermentation liquid by activated sludge submitted to the aerobic feeding and discharge process. Environ Sci Technol 43: 7734-7741.

Johnston B, Jiang G, Hill D, Adamus G, Kwiecień I, Zięba M, Radecka I (2017) The molecular level characterization of biodegradable polymers originated from polyethylene using non-oxygenated polyethylene wax as a carbon source for polyhydroxyalkanoate production. Bioengineering 4: 73 .

Jossek R, Reichelt R, Steinbüchel A (1998) In vitro biosynthesis of poly (3-hydroxybutyric acid) by using purified poly (hydroxyalkanoic acid) synthase of Chromatium vinosum. Appl Microbiol Biot 49: 258-266.

Jurasek L, Marchessault RH (2004) Polyhydroxyalkanoate (PHA) granule formation in Ralstonia eutropha cells: a computer simulation. Appl Microbiol Biot 64: 611-617.

Kadouri D, Jurkevitch E, Okon Y, Castro-Sowinski S (2005) Ecological and agricultural significance of bacterial polyhydroxyalkanoates. Crit Rev Microbiol 31: 55-67.

Kai Z, Ying D, Guo-Qiang C (2003) Effects of surface morphology on the biocompatibility of polyhydroxyalkanoates. Biochem Eng J 16: 115-123.

Kawaguchi Y, Doi Y (1992) Kinetics and mechanism of synthesis and degradation of poly (3-hydroxybutyrate) in Alcaligenes eutrophus. Macromolecules 25: 2324-2329.

Kim BS, Chang HN (1995) Control of glucose feeding using exit gas data and its application to the production of PHB from tapioca hydrolysate by Alcaligenes eutrophus. Biotechnol Tech 9:311-314.

Kim BS, Chang HN (1998) Production of poly (3-hydroxybutyrate) from starch by Azotobacter chroococcum. Biotechnol Lett 20: 109-112.

Kim BS, Lee SC, Lee SY, Chang HN, Chang YK, Woo SI (1994) Production of poly (3-hydroxybutyric acid) by fed-batch culture of Alcaligenes eutrophus with glucose concentration control. Biotechnol Bioeng 43: 892-898.

Kim BS, Lee SY, Chang HN (1992) Production of poly- $\beta$-hydroxybutyrate by fed-batch culture of recombinant Escherichia coli. Biotechnol Lett 14: 811-816. 
Kim GJ, Lee IY, Yoon SC, Shin YC, Park YH (1997) Enhanced yield and a high production of medium-chain-length poly (3-hydroxyalkanoates) in a two-step fed-batch cultivation of Pseudomonas putida by combined use of glucose and octanoate. Enzyme Microb Tech 20: 500-505.

Koller M (2020) Advances in Polyhydroxyalkanoate (PHA) production. Bioengineering. doi: 10,3390/bioengineering7010024.

Koller M, Bona R, Chiellini E, Fernandes EG, Horvat P, Kutschera C, Hesse P, Braunegg G (2008) Polyhydroxyalkanoate production from whey by Pseudomonas hydrogenovora. Bioresour Technol 99: 4854-4863.

Koller M, Bona R, Hermann C, Horvat P, Martinz J, Neto J, Pereira L, Varila P, Braunegg G (2005) Biotechnological production of poly (3-hydroxybutyrate) with Wautersia eutropha by application of green grass juice and silage juice as additional complex substrates. Biocatal Biotransfor 23: 329-337.

Koller M, Maršálek L, de Sousa Dias MM, Braunegg G (2017) Producing microbial polyhydroxyalkanoate (PHA) biopolyesters in a sustainable manner. New Biotechnol 37: 24-38.

Koyama N, Doi Y (1995) Continuous production of poly (3-hydroxybutyrate-co-3-hyhroxyvalerate) by Alcaligenes eutrophus. Biotechnol Lett 17: 281-284.

Koyama N, Doi Y (1997) Miscibility of binary blends of poly [(R)-3-hydroxybutyric acid] and poly [(S)-lactic acid] Polymer 38: 1589-1593.

Kurja J, Zirkzee HF, De Koning GM, Maxwell IA (1995) A new kinetic model for the accumulation of poly (3-hydroxybutyrate) in Alcaligenes eutrophus, 1. Granule growth. Macromol Theor Simul 4: 839-855.

Kusaka S, Abe H, Lee SY, Doi Y (1997) Molecular mass of poly [(R)-3-hydroxybutyric acid] produced in a recombinant Escherichia coli. Appl Microbiol Biot 47: 140-143.

Lai SM, Sun WW, Don TM (2015) Preparation and characterization of biodegradable polymer blends from poly (3-hydroxybutyrate)/poly (vinyl acetate)-modified corn starch. Polym Eng Sci 55: 1321-1329.

Lawrence AG, Choi J, Rha C, Stubbe J, Sinskey AJ (2005) In Vitro Analysis of the Chain Termination Reaction in the Synthesis of Poly-(R)- $\beta$-hydroxybutyrate by the Class III Synthase from Allochromatium vinosum. Biomacromolecules 6: 2113-2119.

Lee SY (1996) Plastic bacteria? Progress and prospects for polyhydroxyalkanoate production in bacteria. Trends Biotechnol 14: 431-438.

Lemoigne M (1926) Products of dehydration and of polymerization of B-hydroxybutyric acid. Bull Soc Chem Biol 8: 770-782.

Lim J, Chong MSK, Teo EY, Chen GQ, Chan JK, Teoh SH (2013) Biocompatibility studies and characterization of poly (3-hydroxybutyrate-co-3-hydroxyhexanoate)/polycaprolactone blends. J Biomed Mater Res B 101: 752-761.

Liu Q, Luo G, Zhou XR, Chen GQ (2011) Biosynthesis of poly (3-hydroxydecanoate) and 3-hydroxydodecanoate dominating polyhydroxyalkanoates by $\beta$-oxidation pathway inhibited $P$ seudomonas putida. Metab Eng 13:11-17.

Madden LA, Anderson AJ, Shah DT, Asrar J (1999) Chain termination in polyhydroxyalkanoate synthesis: involvement of exogenous hydroxy-compounds as chain transfer agents. Int J Biol Macromol 25: 43-53.

Madison LL, Huisman GW (1999) Metabolic engineering of poly (3-hydroxyalkanoates): from DNA to plastic. Microbiol Mol Biol R 63: 21-53.

Marangoni C, Furigo Jr A, de Aragão GM (2002) Production of poly (3-hydroxybutyrate-co-3-hydroxyvalerate) by Ralstonia eutropha in whey and inverted sugar with propionic acid feeding. Process Biochem 38: 137-141.
Marchessault RH (1996) Tender morsels for bacteria: recent development in microbial polyester. Trends Polym Sci 4: 163-168.

Moita R, Freches A, Lemos PC (2014) Crude glycerol as feedstock for polyhydroxyalkanoates production by mixed microbial cultures. Water Res 58: 9-20.

Mousavioun P, Doherty WO, George G (2010) Thermal stability and miscibility of poly (hydroxybutyrate) and soda lignin blends. Ind Crop Prod 32: 656-661.

Mousavioun P, George GA, Doherty WO (2012) Environmental degradation of lignin/poly (hydroxybutyrate) blends. Polym Degrad Stabil 97: 1114-1122.

Mousavioun P, Halley PJ, Doherty WO (2013) Thermophysical properties and rheology of $\mathrm{PHB} /$ lignin blends. Ind Crop Prod 50: 270-275.

Narodoslawsky M, Shazad K, Kollmann R, Schnitzer H (2015) LCA of PHA production-Identifying the ecological potential of bio-plastic. Chem Biochem Eng Q 29: 299-305.

Nawrath C, Poirier Y, Somerville C (1994) Targeting of the polyhydroxybutyrate biosynthetic pathway to the plastids of Arabidopsis thaliana results in high levels of polymer accumulation. P Natl Acad Sci 91: 12760-12764.

Nikel PI, de Almeida A, Melillo EC, Galvagno MA, Pettinari MJ (2006) New recombinant Escherichia coli strain tailored for the production of poly (3-hydroxybutyrate) from agro-industrial by-products. Appl Environ Microb 72: 3949-3954.

Nikodinovic-Runic J, Flanagan M, Hume AR, Cagney G, O'Connor KE (2009) Analysis of the Pseudomonas putida CA-3 proteome during growth on styrene under nitrogen-limiting and non-limiting conditions. Microbiology 155: 3348-3361.

Nobes GA, Jurasek L, Marchessault RH, Martin DP, Putaux JL, Chanzy H (2000) Growth and kinetics of in vitro poly ([R]-(-)-3-hydroxybutyrate) granules interpreted as particulate polymerization with coalescence. Macromol Rapid Commun 21: 77-84.

Noda I, Satkowski MM, Dowrey AE, Marcott C (2004) Polymer alloys of Nodax copolymers and poly (lactic acid). Macromol Biosci 4: 269-275.

Oeding V, Schlegel HG (1973) Beta-ketothiolase from Hydrogenomonas eutropha HI6 and its significance in the regulation of polybeta-hydroxybutyrate metabolism. Biochem J 134: 239248.

Ohkoshi I, Abe H, Doi Y (2000) Miscibility and solid-state structures for blends of poly [(S)-lactide] with atactic poly [(R S)-3-hydroxybutyrate]. Polymer 41: 5985-5992.

Ojumu TV, Yu J, Solomon BO (2004) Production of polyhydroxyalkanoates a bacterial biodegradable polymer. Afr J Biotechnol 3: $18-24$.

Pilkington SM, Encke B, Krohn N, Hoehne M, Stitt M, Pyl ET (2015) Relationship between starch degradation and carbon demand for maintenance and growth in Arabidopsis thaliana in different irradiance and temperature regimes. Plant Cell Environ 38: 157-171.

Povolo S, Toffano P, Basaglia M, Casella S (2010) Polyhydroxyalkanoates production by engineered Cupriavidus necator from waste material containing lactose. Bioresour Technol 101: 7902-7907.

Pozo C, Martnez-Toledo MV, Rodelas B, Gonzalez-Lopez J (2002) Effects of culture conditions on the production of polyhydroxyalkanoates by Azotobacter chroococcum $\mathrm{H} 23$ in media containing a high concentration of alpechn (wastewater from olive oil mills) as primary carbon source. J Biotechnol 97: 125-131.

Preusting H, Kingma J, Huisman G, Steinbüchel A, Witholt B (1993) Formation of polyester blends by a recombinant strain of Pseudomonas oleovorans: Different poly(3-hydroxyal- 
kanoates) are stored in separate granules. J Environ Polym Degrad 1: 11-21.

Preusting HA, Nijenhuis, Witholt B (1990) Physical characteristics of poly(3-hydroxyalkanoates) and poly(3-hydroxyalkanoates) produced by Pseudomonas oleovorans grown on aliphatichydrocarbons. Macromolecules 23: 4220-4224.

Reddy CSK, Ghai R, Kalia V (2003) Polyhydroxyalkanoates: an overview. Bioresour Technol 87: 137-146.

Rhee YH, Jang JH, Rogers PL (1993) Production of copolymer consisting of 3-hydroxybutyrate and 3-hydroxyvalerate by fed-batch culture of Alcaligenes sp. SH-69. Biotechnol Lett 15: 377-382.

Ribera RG, Monteoliva-Sanchez M, Ramos-Cormenzana A (2001) Production of polyhydroxyalkanoates by Pseudomonas putida KT2442 harboring pSK2665 in wastewater from olive oil mills (alpechin). Electron J Biotechn 4: 11-12.

Saad GR (2002) Blends of bacterial poly [(R)-3-hydroxybutyrate] with oligo [(R S)-3-hydroxybutyrate]-diol. Polym Int 51: 338348 ,

Satoh Y, Minamoto N, Tajima K, Munekata M (2002) Polyhydroxyalkanoate synthase from Bacillus sp, INT005 is composed of PhaC and PhaR. J Biosci Bioeng 94: 343-350.

Schubert P, Steinbüchel A, Schlegel HG (1988) Cloning of the Alcaligenes eutrophus genes for synthesis of poly-beta-hydroxybutyric acid (PHB) and synthesis of PHB in Escherichia coli. J Bacteriol 170: 5837-5847.

Senior PJ, Beech GA, Ritchie GAF, Dawes EA (1972) The role of oxygen limitation in the formation of poly- $\beta$-hydroxybutyrate during batch and continuous culture of Azotobacter beijerinckii. Biochem J 128: 1193-1201, doi: 10.1042/bj1281193.

Shi F, Gross RA, Rutherford DR (1996) Microbial polyester synthesis: effects of poly (ethylene glycol) on product composition repeat unit sequence and end group structure. Macromolecules 29: $10-17$.

Silva LF, Taciro MK, Ramos MM, Carter JM, Pradella JGC, Gomez JGC (2004) Poly-3-hydroxybutyrate (P3HB) production by bacteria from xylose glucose and sugarcane bagasse hydrolysate. J Ind Microbiol Biot 31: 245-254.

Slater SC, Voige WH, Dennis DE (1988) Cloning and expression in Escherichia coli of the Alcaligenes eutrophus H16 poly-beta-hydroxybutyrate biosynthetic pathway. J Bacteriol 170: 4431-4436.

Snell KD, Peoples OP (2002) Polyhydroxyalkanoate polymers and their production in transgenic plants. Metab Eng 4: 29-40.

Solaiman DK, Ashby RD, Hotchkiss AT, Foglia TA (2006) Biosynthesis of medium-chain-length poly (hydroxyalkanoates) from soy molasses. Biotechnol Lett 28: 157-162.

Stal LJ (1992) Poly (hydroxyalkanoate) in cyanobacteria: an overview. FEMS Microbiol Lett 103: 169-180.

Stavroula K, Simos M, Joanne HK (2020) Polyhydroxyalkanoates (PHAs) from Household Food Waste: Research Over the Last Decade. Int J Biotech and Bioeng 6: 26-36.

Steinbuchel A (1991) Polyhydroxyalkanoic acids. In: Byrom D (ed) Biomaterials: Novel Materials from Biological Sources. Stockton Press, New York, pp 124-213.

Steinbuchel A, Fuchtenbusch B (1998) Bacteria and other biological systems for polyester production. TIBTECH 16: 419-427.

Steinbüchel A, Schlegel HG (1991) Physiology and molecular genetics of poly ( $\beta$-hydroxyalkanoic acid) synthesis in Alcaligenes eutrophus. Mol Microbiol 5: 535-542.

Steinbuchel A, Lutke-Eversloh T (2003) Metabolic engineering and pathway construction for biotechnological production of relevant polyhydroxyalkanoates in microorganisms. Biochem Eng J 16: 81-96.
Stubbe J, Tian J (2003) Polyhydroxyalkanoate (PHA) homeostasis: the role of the PHA synthase. Nat Prod Rep 20: 445-457.

Stubbe J, Tian J, He A, Sinskey AJ, Lawrence AG, Liu P (2005) Nontemplate-dependent polymerization processes: polyhydroxyalkanoate synthases as a paradigm. Annu Rev Biochem 74: 433-480

Sudesh K, Abe H, Doi Y (2000) Synthesis structure and properties of polyhydroxyalkanoates: biological polyesters. Prog Polym Sci 25: 1503-1555.

Sun Z, Ramsay JA, Guay M, Ramsay B (2007) Increasing the yield of MCL-PHA from nonanoic acid by co-feeding glucose during the PHA accumulation stage in two-stage fed-batch fermentations of Pseudomonas putida KT2440. J Biotechnol 132: 280-282.

Suriyamongkol P, Weselake R, Narine S, Moloney M, Shah S (2007) Biotechnological approaches for the production of polyhydroxyalkanoates in microorganisms and plants - a review. Biotechnol Adv 25: 148-175.

Taguchi S, Doi Y (2004) Evolution of polyhydroxyalkanoate (PHA) production system by "enzyme evolution": successful case studies of directed evolution. Macromol Biosci 4: 145-156.

Takagi Y, Yasuda R, Yamaoka M, Yamane T (2004) Morphologies and mechanical properties of polylactide blends with medium chain length poly (3-hydroxyalkanoate) and chemically modified poly (3-hydroxyalkanoate). J Appl Polym Sci 93: 2363-2369.

Takase K, Matsumoto KI, Taguchi S, Doi Y (2004) Alteration of substrate chain-length specificity of type II synthase for polyhydroxyalkanoate biosynthesis by in vitro evolution: in vivo and in vitro enzyme assays. Biomacromolecules 5: 480-485.

Tian J, He A, Lawrence AG, Liu P, Watson N, Sinskey AJ, Stubbe J (2005) Analysis of transient polyhydroxybutyrate production in Wautersia eutropha $\mathrm{H} 16$ by quantitative Western analysis and transmission electron microscopy. J Bacteriol 187: 3825-3832.

Tsuge T (2002) Metabolic improvements and use of inexpensive carbon sources in microbial production of polyhydroxyalkanoates. J Biosci Bioeng 94: 579-584.

Valappil SP, Peiris D, Langley GJ, Herniman JM, Boccaccini AR, Bucke C, Roy I (2007) Polyhydroxyalkanoate (PHA) biosynthesis from structurally unrelated carbon sources by a newly characterized Bacillus spp. J Biotechnol 127: 475-487.

Van-Thuoc D, Quillaguaman J, Mamo G, Mattiasson B (2008) Utilization of agricultural residues for poly (3-hydroxybutyrate) production by Halomonas boliviensis LC1. J Appl Microbiol 104: 420-428.

Verlinden RA, Hill DJ, Kenward MA, Williams CD, Radecka I (2007) Bacterial synthesis of biodegradable polyhydroxyalkanoates. J Appl Microbiol 102: 1437-1449.

Wang C, Venditti RA, Zhang K (2015) Tailor-made functional surfaces based on cellulose-derived materials. Appl Microbiol Biot 99: 5791-5799.

Wang JG, Bakken LR (1998) Screening of soil bacteria for polybeta-hydroxybutyric acid production and its role in the survival of starvation. Microb Ecol 35: 94-101.

Weihua K, He Y, Asakawa N, Inoue Y (2004) Effect of lignin particles as a nucleating agent on crystallization of poly (3-hydroxybutyrate). J Appl Polym Sci 94: 2466-2474.

Willett JL, Kotnis MA, O'brien GS, Fanta GF, Gordon SH (1998) Properties of starch-graft-poly (glycidyl methacrylate)-PHBV composites. J Appl Polym Sci 70: 1121-1127.

Witholt B, Kessler B (1999) Perspectives of medium chain length poly (hydroxyalkanoates) a versatile set of bacterial bioplastics. Curr Opin Biotech 10: 279-285.

Yalpani M, Marchessault RH, Morin FG, Monasterios CJ (1991) Synthesis of poly (3-hydroxyalkanoate) (PHA) conjugates: 
PHA-carbohydrate and PHA-synthetic polymer conjugates. Macromolecules 24: 6046-6049.

Yao H, Wu LP, Chen GQ (2019) Synthesis and characterization of electro-conductive PHA-graft-graphene nanocomposites. Biomacromolecules 2: 645-652.

York GM, Stubbe J, Sinskey AJ (2002) The Ralstonia eutropha PhaR protein couples synthesis of the PhaP phasin to the presence of polyhydroxybutyrate in cells and promotes polyhydroxybutyrate production. J Bacteriol Res 184: 59-66.

Yoshie N, Saito M, Inoue Y (2004) Effect of chemical compositional distribution on solid-state structures and properties of poly (3-hydroxybutyrate-co-3-hydroxyvalerate). Polymer 45: 1903-1911.

Yuan H, Chen Y, Zhang H, Jiang S, Zhou Q, Gu G (2006) Improved bioproduction of short-chain fatty acids (SCFAs) from excess sludge under alkaline conditions. Environ Sci Technol 40: 2025-2029.

Yuan W, Jia Y, Tian J, Snell KD, Müh U, Sinskey AJ, Lambalot RH, Walsh CT, Stubbe J (2001) Class I and III polyhydroxyalkanoate synthases from Ralstonia eutropha and Allochromatium vino- sum: characterization and substrate specificity studies. Arch Biochem Biophys 394: 87-98.

Zhang H, Obias V, Gonyer K, Dennis D (1994) Production of polyhydroxyalkanoates in sucrose-utilizing recombinant Escherichia coli and Klebsiella strains. Appl Environ Microb 60: 1198-1205.

Zhang L, Deng X, Huang Z (1997a) Miscibility thermal behaviour and morphological structure of poly (3-hydroxybutyrate) and ethyl cellulose binary blends. Polymer 38: 5379-5387.

Zhang L, Deng X, Zhao S, Huang Z (1997b) Biodegradable polymer blends of poly (3-hydroxybutyrate) and starch acetate. Polym Int 44: 104-110.

Zhang L, Xiong C, Deng X (1996) Miscibility crystallization and morphology of poly ( $\beta$-hydroxybutyrate)/poly (d l-lactide) blends. Polymer 37: 235-241.

Zinn M, Weilenmann HU, Hany R, Schmid M, Egli TH (2003) Tailored Synthesis of Poly ([R]-3-hydroxybutyrate-co-3-hydroxyvalerate) (PHB/HV) in Ralstonia eutropha DSM 428. Eng Life Sci 23: 309-316. 